\title{
Lipidomics of human umbilical cord serum: identification of unique sterol sulfates
}

\begin{abstract}
Aim: There are currently limited lipidomics data for human umbilical cord blood. Therefore, the lipidomes of cord sera from six newborns and sera from six nonpregnant females were compared. Materials \& methods: Sera lipidomics analyses were conducted using a high-resolution mass spectrometry analytical platform. Results: Cord serum contained a diverse array of glycerophospholipids, albeit generally at lower concentrations than monitored in adult serum. The unexpected observations were that cord serum contained several neurosteroid sulfates and bile acid sulfates that were not detectable in adult serum. Conclusion: Our data are the first to demonstrate that cord serum contains bile acid sulfates that are synthesized early in the hydroxylase, neutral and acidic pathways of primary bile acid biosynthesis and support previous publications of cord blood perfluoralkyl toxins in newborns.
\end{abstract}

Lay abstract: Umbilical cord blood offers the potential to increase our understanding of fetal development during pregnancy and during development after delivery. Our studies of complex sterols in umbilical cord blood (bile acid sulfates) suggest that with further studies these may be useful biomarkers of abnormal fetal liver development.

First draft submitted: 17 January 2017; Accepted for publication: 24 February 2017; Published online: 5 April 2017

Keywords: bile acid sulfates $\bullet$ glycerophospholipids $\bullet$ lipidomics $\bullet$ neurosteroid sulfates - perfluoroalkyl toxins $\bullet$ umbilical cord serum

The vessels of the umbilical cord supply the fetus with oxygen and nutrients, and remove fetal waste products. Even though percutaneous umbilical cord blood sampling has been an established method for obtaining samples for fetal genetic testing during the last decades, limited evaluation of umbilical cord blood lipidomics has been undertaken thus far $[1,2]$. To define the lipidomics differences between the lipid profiles of cord sera and adult female sera, we undertook a nontargeted pilot study of cord lipidomics utilizing a high-resolution mass spectrometric platform. This involved an evaluation of cord sera from six healthy newborns and compared their lipid profiles with those of the healthy nonpregnant adult females. Nonpregnant adult females were chosen as the controls so that the baseline measurements were not complicated by the fluid communications between the fetus and a pregnant mother. Our data add to the human cord blood lipidomics database covering glycerophospholipids, sphingolipids, neutral lipids and free fatty acids.

Cord serum contained a diverse array of glycerophospholipids, albeit generally at lower concentrations than monitored in adult serum. No major differences in the diversity or levels of sphingolipids were detected. In contrast, cord serum levels of neutral lipids (diacylglycerols [DAG] and triacylglycerols [TAG]) were dramatically lower than adult serum. Levels of free fatty acids were similar. We were also able to describe for the first time that cord serum contains levels of bile acid sulfates not detected in adult serum.
Paul L Wood ${ }^{*, 1,2}$, Heli Siljander ${ }^{3,4}$ \& Mikael Knip K $^{3,4,6}$ 'Metabolomics Unit, Department of Physiology \& Pharmacology, DeBusk College of Osteopathic Medicine, TN, USA

${ }^{2}$ College of Veterinary Medicine, Lincoln Memorial University, 6965 Cumberland Gap Pkwy., Harrogate, TN 37752, USA ${ }^{3}$ Children's Hospital, University of Helsinki \& Helsinki University Hospital, Helsinki, Finland

${ }^{4}$ Research Programs Unit, Diabetes \& Obesity, University of Helsinki, Helsinki, Finland

${ }^{5}$ Folkhälsan Research Center, University of Helsinki, Helsinki, Finland

${ }^{6}$ Department of Pediatrics, Tampere University Hospital, Tampere, Finland *Author for correspondence: Tel.: +1 4238696666 paul.wood@Imunet.edu 


\section{Materials \& methods}

Blood collection \& processing

Umbilical cord arterial blood was collected at parturition, according to an Ethics Committee-approved protocol. Serum was collected and frozen at $-80^{\circ} \mathrm{C}$ prior to sample processing. Blood was also collected for three male and three female newborn infants.
Control adult female serum was collected from six nonpregnant women (age 32-40 years).

\section{Lipidomics analyses of serum}

Lipid profiles were analyzed for all serum samples as follows. After thawing, $100 \mu \mathrm{l}$ aliquots of serum were mixed with $1 \mathrm{ml}$ of methanol containing stable-isotope internal
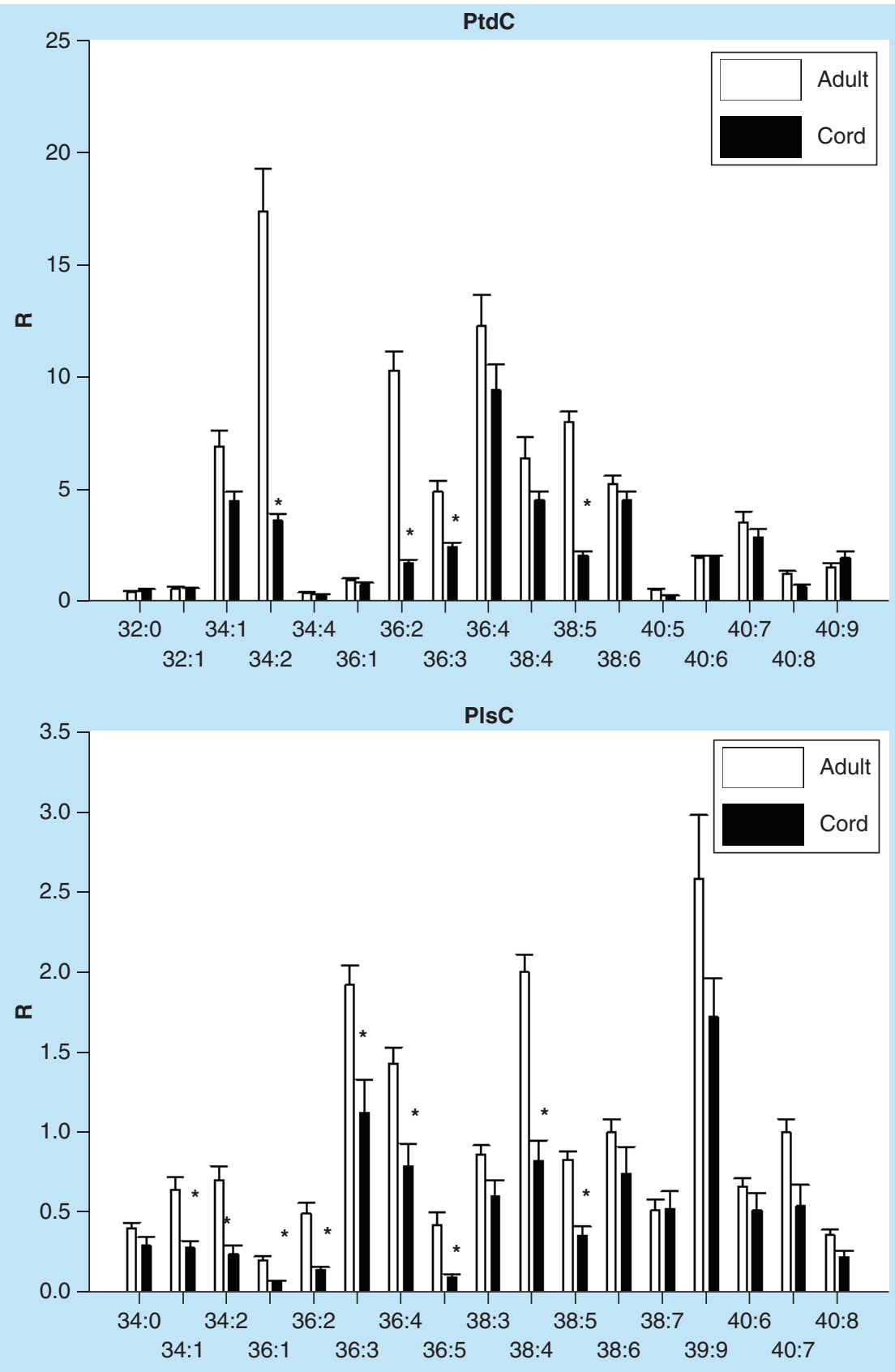

Figure 1. Umbilical cord serum levels of phosphatidylcholines and choline plasmalogens. Mean \pm SD. The nomenclature refers to the fatty acid or fatty alcohol substitutions at sn-1 and sn-2 of the glycerol backbone. For example, 32:1 refers to 32 carbons with one double bond.

${ }^{*} \mathrm{p}<0.01$.

PlsC: Choline plasmalogen; PtdC: Phosphatidylcholine; R: Ratio of peak area of endogenous lipid to the peak area of the internal standard. 

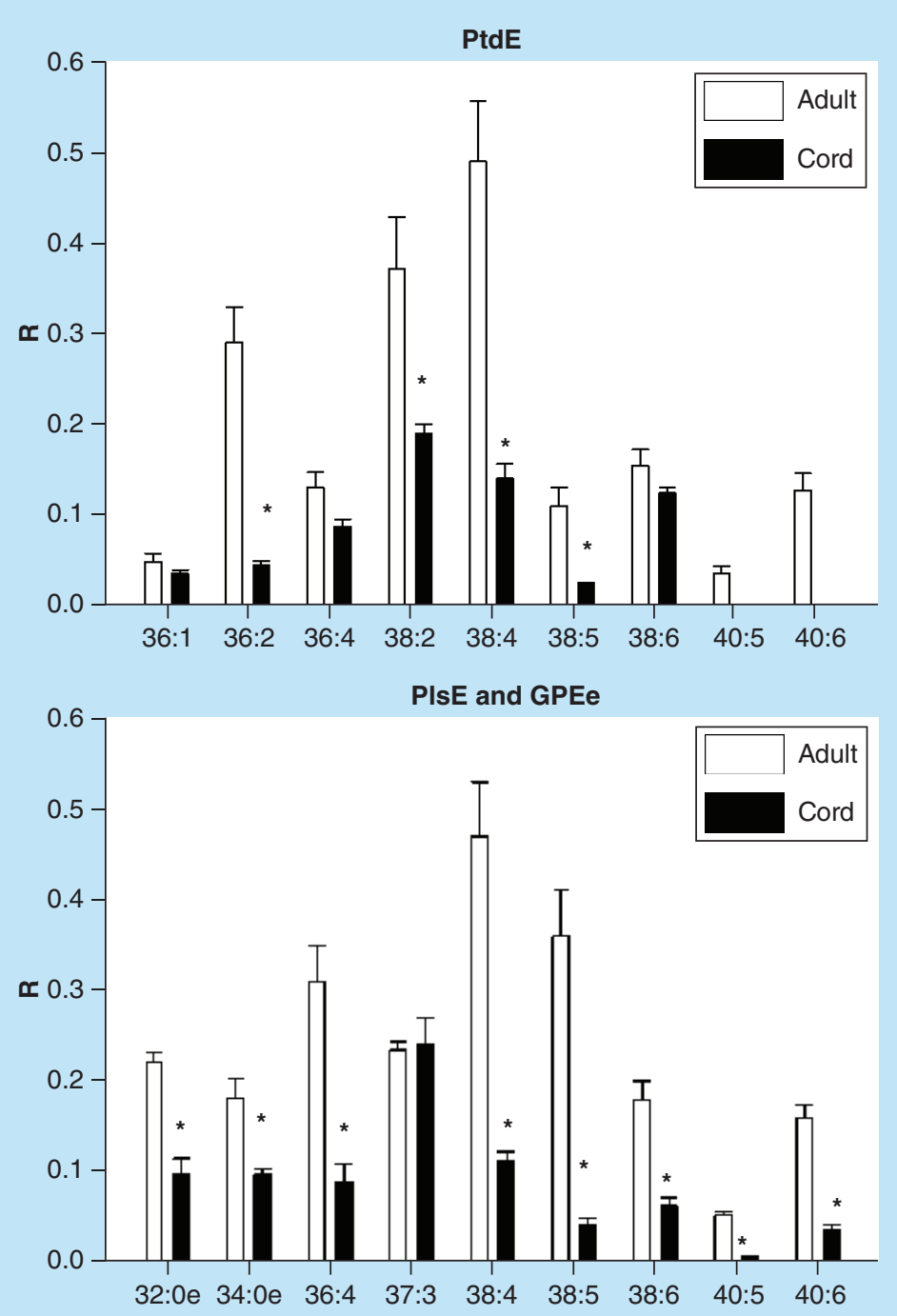

Figure 2. Umbilical cord serum levels of phosphatidylethanolamines, ethanolamine plasmalogens and 1-alkyl-2-acyl glycerophosphoethanolamines. Mean \pm SD. The nomenclature refers to the fatty acid or fatty alcohol substitutions at sn-1 and sn-2 of the glycerol backbone. For example, 36:4 refers to 36 carbons with four double bonds. ${ }^{*} \mathrm{p}<0.01$.

GPEe: 1-alkyl-2-acyl glycerophosphoethanolamine; PIsE: Ethanolamine plasmalogen;

PtdE: Phosphatidylethanolamine; R: ratio of peak area of endogenous lipid to the peak area of the internal standard.

standards [3,4]. Next, $1 \mathrm{ml}$ of water and $2 \mathrm{ml}$ of methyltert-butyl ether were added and the tubes were vigorously shaken at room temperature for $30 \mathrm{~min}$. After centrifugation at $3000 \times \mathrm{g}$ for $10 \mathrm{~min}, 1 \mathrm{ml}$ of the upper organic layer was dried by centrifugal vacuum evaporation prior to dissolution in $150 \mu \mathrm{l}$ of isopropanol:methanol:chloroform (4:2:1) containing $15 \mathrm{mM}$ ammonium acetate. Shotgun ESI lipidomics $(5 \mu \mathrm{l} / \mathrm{min})$ was performed utilizing high-resolution data acquisition (140,000 at $200 \mathrm{amu}$; $0.3-3$ ppm mass error; $m / z 200-1400$ ) with an orbitrap mass spectrometer (Thermo Q Exactive).

In negative-ion ESI ( $3.2 \mathrm{kV}$, capillary temperature of $320^{\circ} \mathrm{C}$, sheath gas of 10 ), the anions of ethanolamine plasmalogens (PlsEtn), phosphatidylglycerols (PG), phosphatidylinositols (PtdIn), sterol sulfates, free fatty acids and perfluoroalkyl toxins, and the $[\mathrm{M}+\mathrm{HCOO}]^{-}$ anions of ceramides were monitored. In positive-ion ESI ( $4.3 \mathrm{kV}$, capillary temperature of $320^{\circ} \mathrm{C}$, sheath gas of 10), the cations of choline plasmalogens $(\mathrm{PlsCh})$, phosphatidylcholines, ceramides and sphingomyelins (SM) and the ammonium adducts of DAG and TAG were monitored.

Data are presented as $R$ values (ratio of the endogenous lipid peak area to the peak area of an appropriate internal standard) per $100 \mu \mathrm{l}$ of cord or adult serum $(n=6)$. 


\section{Identification of unknowns}

The exact masses of unknown metabolites were used to interrogate public databases for compound identification. These databases included LipidMaps, hmdb and Kegg. Putative metabolites were subsequently validated by $\mathrm{MS}^{2}$. Putative aldehyde metabolites were derivatized with Girard's reagent T (GRT). To the dried methyltert-butyl ether extracts, added $0.1 \mathrm{ml}$ of reagent (20 mg GRT per $\mathrm{ml}$ of methanol) and $20 \mu \mathrm{l}$ of acetic acid prior to heating at $70^{\circ} \mathrm{C}$ for $30 \mathrm{~min}$ with shaking. The samples were dried and dissolved in infusion solvent. Aldehydes were augmented in mass by 113.09528 after derivatization with GRT and the $[\mathrm{M}+\mathrm{H}]^{+}$ions of these derivatives were monitored in ESI.

\section{Statistics}

Data were analyzed utilizing the two-tailed t-test assuming unequal variances (Excel).

\section{Results}

\section{Gender differences}

There were no gender differences in the umbilical cord plasma levels of lipids or sterol sulfates between newborn males and females. However, this study had only three samples for each gender.

\section{(O-acyl) $\Omega$-hydroxy-fatty acids}

(O-acyl) $\Omega$-hydroxy-fatty acids, previously detected in amniotic fluid [3], were not detected in human cord blood.
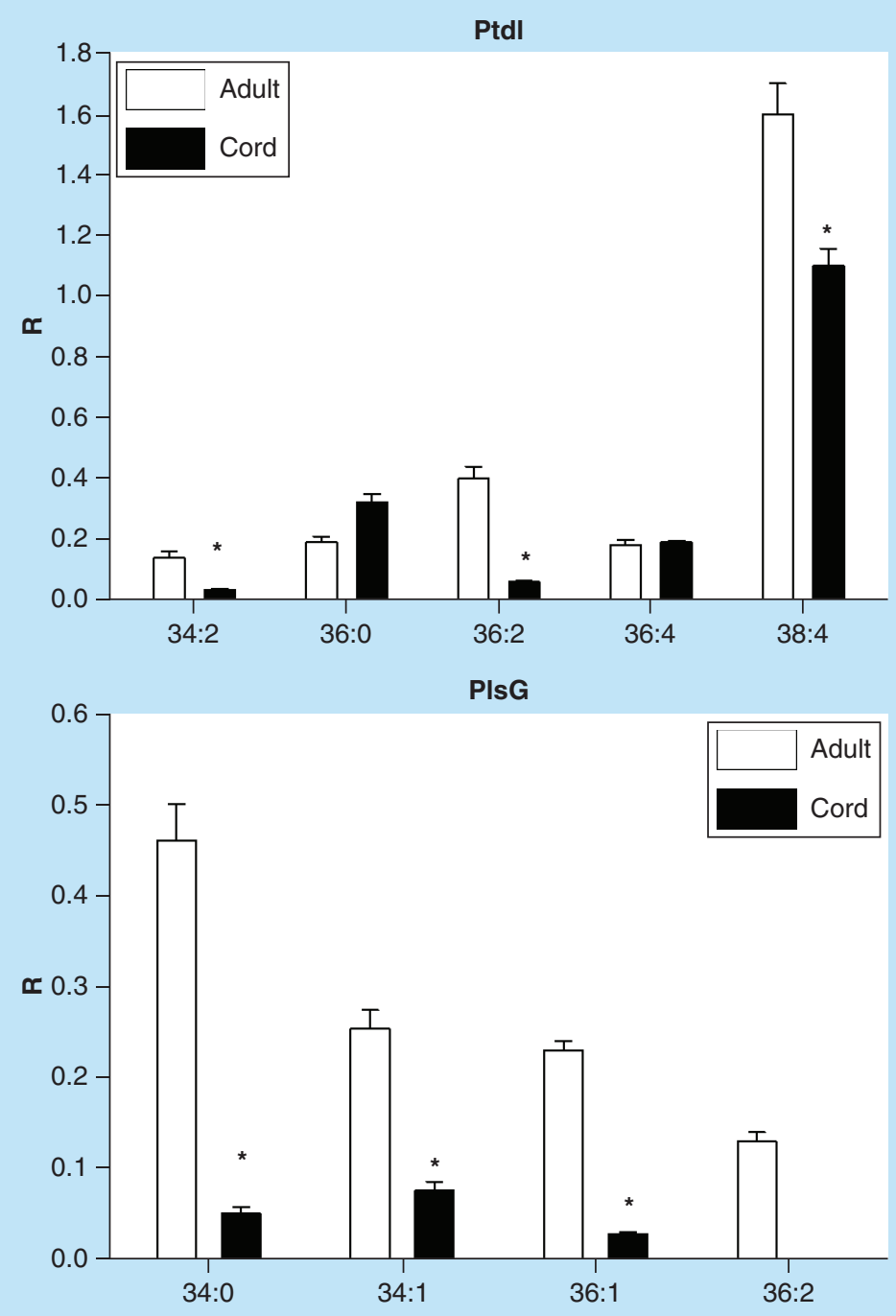

Figure 3. Umbilical cord serum levels of phosphatidylinositols and phosphatidylglycerols. Mean \pm SD. The nomenclature refers to the fatty acid substitutions at sn-1 and sn-2 of the glycerol backbone. For example, 34:3 refers to 34 carbons with three double bonds. ${ }^{*} \mathrm{p}<0.01$.

PtdG: Phosphatidylglycerol; Ptdl: Phosphatidylinositol; R: Ratio of peak area of endogenous lipid to the peak area of the internal standard. 

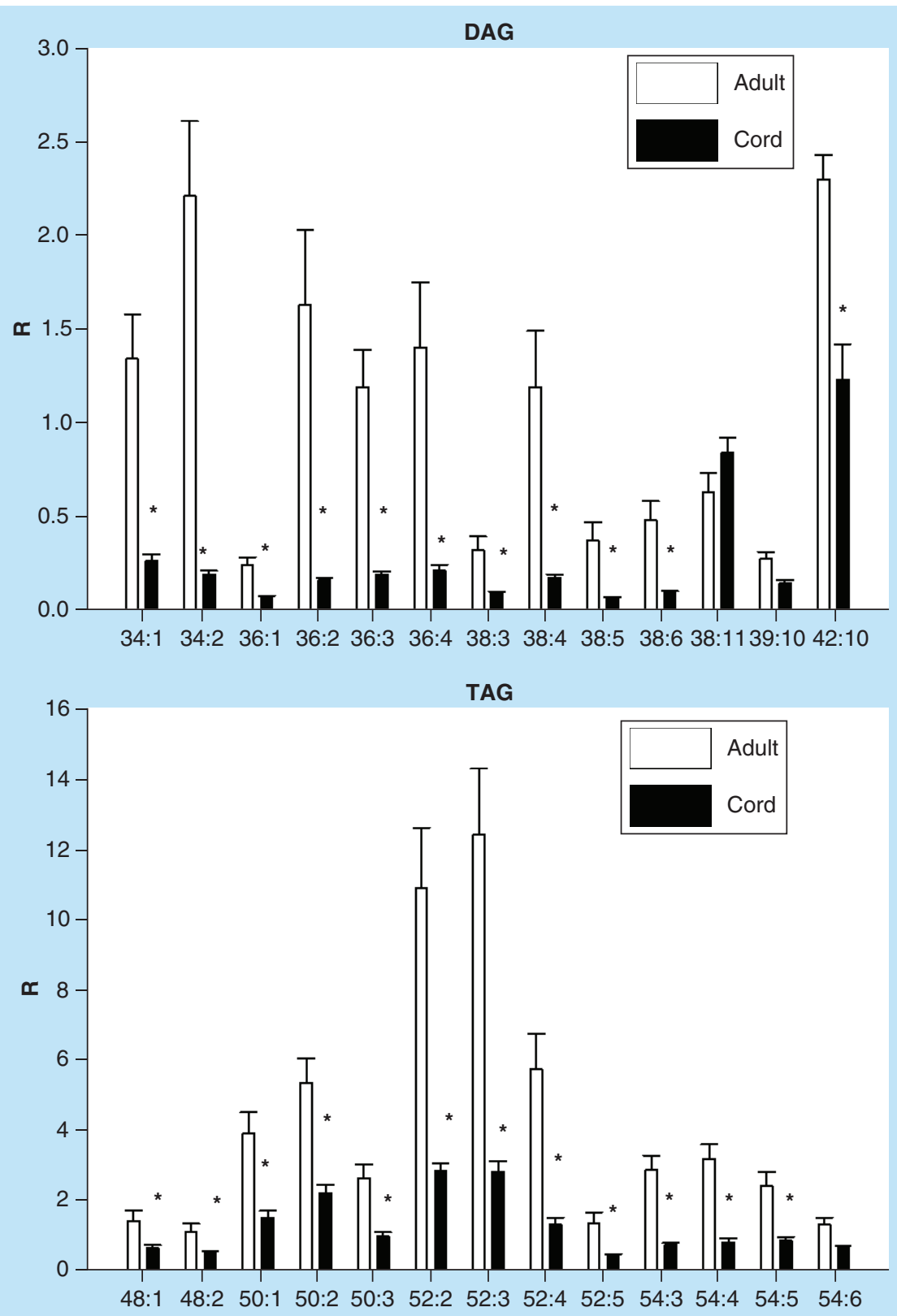

Figure 4. Umbilical cord serum levels of diacylglycerols and triacylglycerols. Mean \pm SD. The nomenclature refers to the fatty acid substitutions of the glycerol backbone. For example, 34:1 refers to 34 carbons with one double bond.

* $p<0.01$.

DAG: Diacylglycerol; R: ratio of peak area of endogenous lipid to the peak area of the internal standard;

TAG: Triacylglycerol.

\section{Choline glycerophospholipids}

Cord serum was observed to possess a similar diversity of diacylglycerophosphocholines (phosphatidylcholines) and $\mathrm{PlsCh}$ as monitored in adult serum (Figure 1). A number of choline glycerophospholipids were monitored at 50-80\% lower levels in cord serum, except for lipids with polyunsaturated fatty acid substitutions with six to nine unsaturated bonds.

\section{Ethanolamine glycerophospholipids}

Phosphatidylethanolamines, alkyl-acyl-phosphatidylethanolamines and PlsE were 50-70\% lower in cord serum than in adult serum (Figure 2).

\section{Phosphatidylinositols \& phosphatidylglycerols}

PtdIn levels were generally lower in cord serum while PG levels were $50-60 \%$ less than measured in adult serum (Figure 3). 
Neutral lipids

DAG and TAG levels were $50-80 \%$ lower in cord serum (Figure 4).

\section{Sphingolipids}

SM and ceramide levels were similar for cord and adult serum with few exceptions showing lower levels in cord blood (Figure 5).

\section{Free fatty acids}

Free fatty acid levels of cord serum were similar to those monitored in adult serum (Figure 6).

\section{Sterol sulfates}

Cord serum was found to contain a diverse array of neurosteroid sulfates not detected in adult serum (Figure 6). These included pregnenolone sulfate, hydroxypregnenolone sulfate, allopregnanolone sulfate and tetrahydrodeoxycorticosterone sulfate. Dehydroepiandrosterone sulfate was detected in both cord and adult plasma, but shotgun lipidomics cannot distinguish dehydroepiandrosterone sulfate from testosterone sulfate.

The bile acids, taurodeoxycholic acid and glycochenodeoxycholic acid sulfate were found at lower levels
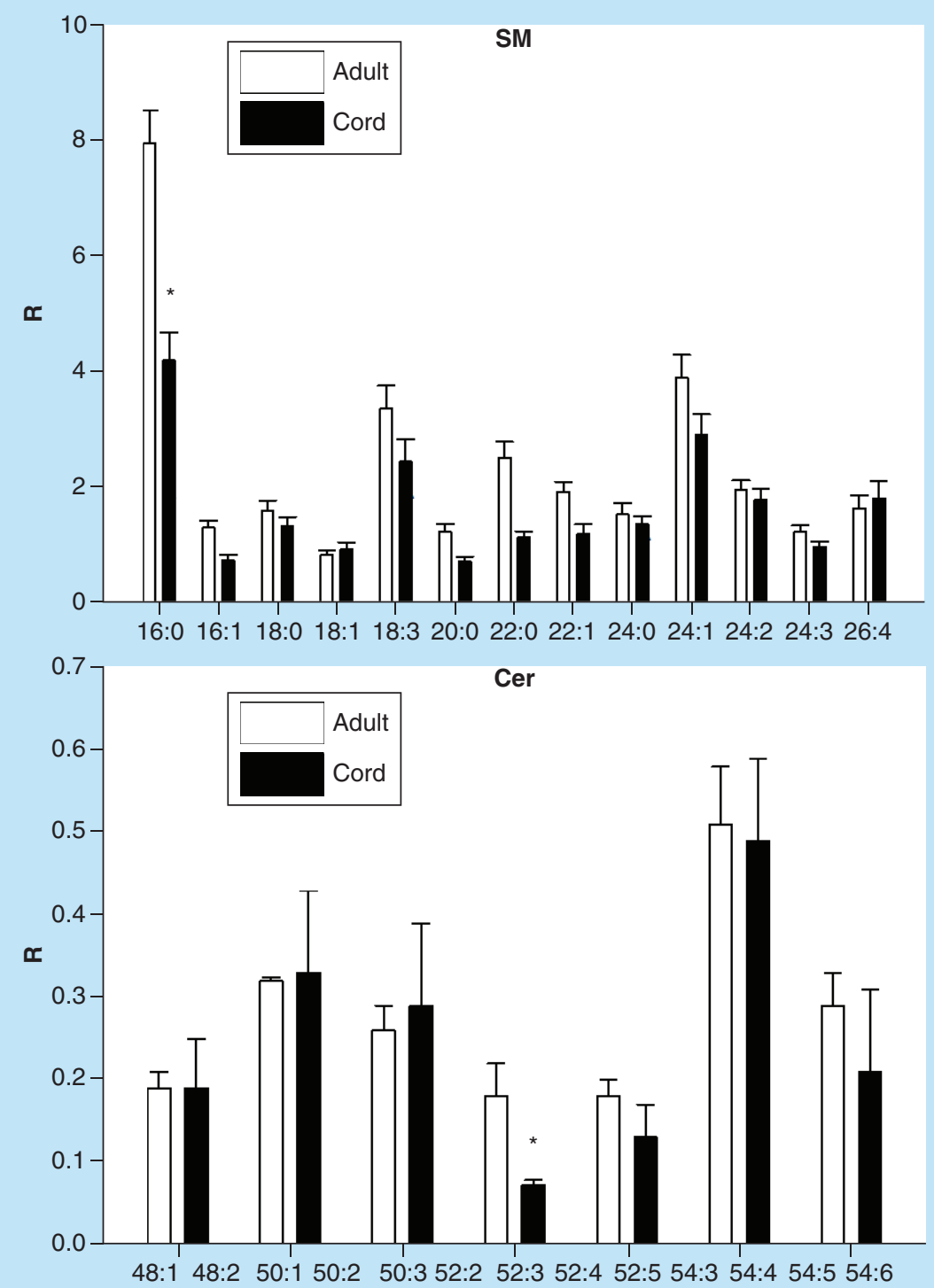

Figure 5. Umbilical cord serum levels of sphingolipids. Mean \pm SD. The nomenclature refers to the fatty acid substitution of the sphingosine backbone. For example, 16:0 refers to palmitic acid.

$* \mathrm{p}<0.01$.

Cer: Ceramide; R: Ratio of peak area of endogenous lipid to the peak area of the internal standard; SM: Sphingomyelin. 
in cord serum than in adult serum. However, several sulfates of bile acid intermediates were only detected in cord serum. $\mathrm{MS}^{2}$ of the sterol sulfates all yielded a strong product anion of $96.9595\left[\mathrm{HSO}_{4}\right]^{-}$with 0.10 $0.21 \mathrm{ppm}$ mass error, supporting that these biomarkers were sulfates. The monitored $[\mathrm{M}-\mathrm{H}]^{-}$anion of 497.2942 could be dihydroxycholesterol sulfate, cholestenetriol sulfate (24 and 25 triol) and/or dihydroxycholestanal sulfate. Derivatization with GRT did not alter the mass of 497.2942, eliminating the possibility that this sterol sulfate was the aldehyde. Therefore, the mass 497.2942 is most likely dihydroxycholesterol sulfate in the acidic pathway and/or cholestenetriol sulfates (24/25 triols) in the hydroxylase pathways of bile acid biosynthesis.

Similarly, the $[\mathrm{M}-\mathrm{H}]^{-}$anion of 513.2892 could be dihydroxycholestanoate sulfate and/or trihydroxycholestanal sulfate. Derivatization with GRT did not alter the mass of 513.2892, eliminating the possibility that this sterol sulfate was the aldehyde. Therefore, the mass 513.2892 is most likely dihydroxycholestanoate sulfate in the neutral pathway of bile acid biosynthesis.

\section{Perfluoroalkyl environmental/industrial toxins}

The industrial toxins and perfluoro-octane sulfonate (PFOS), perfluorononanoic acid, perfluoro-octanoic acid and perfluorohexane sulfonic acid were found in adult serum, while only PFOS and perfluorononanoic acid were detected in cord serum, albeit at much lower concentrations in the case of PFOS (Table 1). $\mathrm{MS}^{2}$ of PFOS and perfluorohexane sulfonic acid yielded a strong product anion of $79.9568\left[\mathrm{SO}_{3}\right]^{-}$with $2.12 \mathrm{ppm}$ mass error, supporting that these were sulfonic acids.

\section{Discussion}

As limited analyses of the umbilical cord serum lipidome have previously been available [1,2], we undertook a detailed lipidomics analysis of cord serum. Unsurprisingly, we found that the umbilical cord serum lipidome is dominated by similar lipids to those circulating in the maternal serum. Our data indicate that in cord sera, the diversities of inositol, choline and ethanolamine glycerophospholipids are comparable to the diversities observed in adult sera, but that a number of these lipids are present at lower levels in the cord blood. Our data are the first to demonstrate the presence of 1-alkyl-2acyl glycerophosphoethanolamines (GPE 32:0e and GPE 34:0e) in cord sera. The lower levels of 1-alkyl2-acyl glycerophosphoethanolamines, $\mathrm{PlsE}$ and $\mathrm{PlsC}$ in cord blood strongly suggest that peroxisomal function, which is responsible for the biosynthesis of ether lipids [5], is active in the fetus [6] and that these vinylether lipids are not exported into the cord blood but are utilized as essential membrane lipids by the fetus.

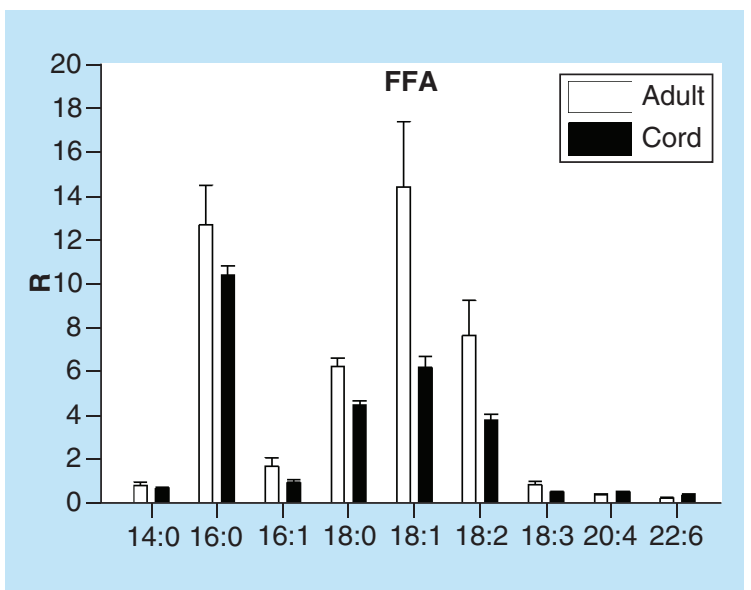

Figure 6. Umbilical cord serum levels of free fatty acids. Mean \pm SD.

$* p<0.01$.

FFA: Free fatty acid; R: Ratio of peak area of endogenous lipid to the peak area of the internal standard.

Cord sera also possessed significantly lower circulating levels of PtdG and PtdI than are present in adult sera. Similarly the neutral lipids (DAG and TAG) were much lower in cord sera. This may indicate that the fetal liver biosynthesis of these lipids is sufficient for fetal metabolism but not for export to the cord blood. Our data also suggest that the fetal liver is the main source of DAG and TAG essential for energy metabolism, signal transduction and biosynthesis of glycerolipids and glycerophospholipids [4].

The levels of free fatty acids and the sphingolipid lipidome profile (levels of SM and ceramides) of the cord sera were comparable to the ones observed in adult sera. These data possibly imply that free fatty acids and sphingolipids may pass freely between maternal and fetal circulations.

Bile acids of the cord sera are generally considered to derive from the fetus rather than the mother $[7,8]$. Polyhydroxylated bile acids and bile acid sulfates are not present in nonpregnant healthy adults, but appear in the serum of patients with inborn errors of bile acid metabolism [8-10] or with cholestatic liver diseases [7]. During pregnancy, polyhydroxylated bile acids produced by the fetal liver are transported via cord blood to the mother for further metabolism and elimination [7,8]. Concentrations of these polyhydroxylated bile acids decrease in healthy infants between the age of 7 years and 30 days postpartum [11]. Our data demonstrate that sulfates of some of these polyhydroxylated bile acids are excreted into the cord blood, presumably via the cations of cytosolic sulfotransferases which catalyze sulfation of hydroxylated sterols [9]. Specifically, sterol sulfates, including cholestenetriol and dihydroxycholestanoate sulfates, were not detected in the sera of the nonpregnant adult females. Elevated levels 
Table 1. Sterol sulfates and environmental perfluoroalkyl toxins monitored in adult and umbilical cord serum.

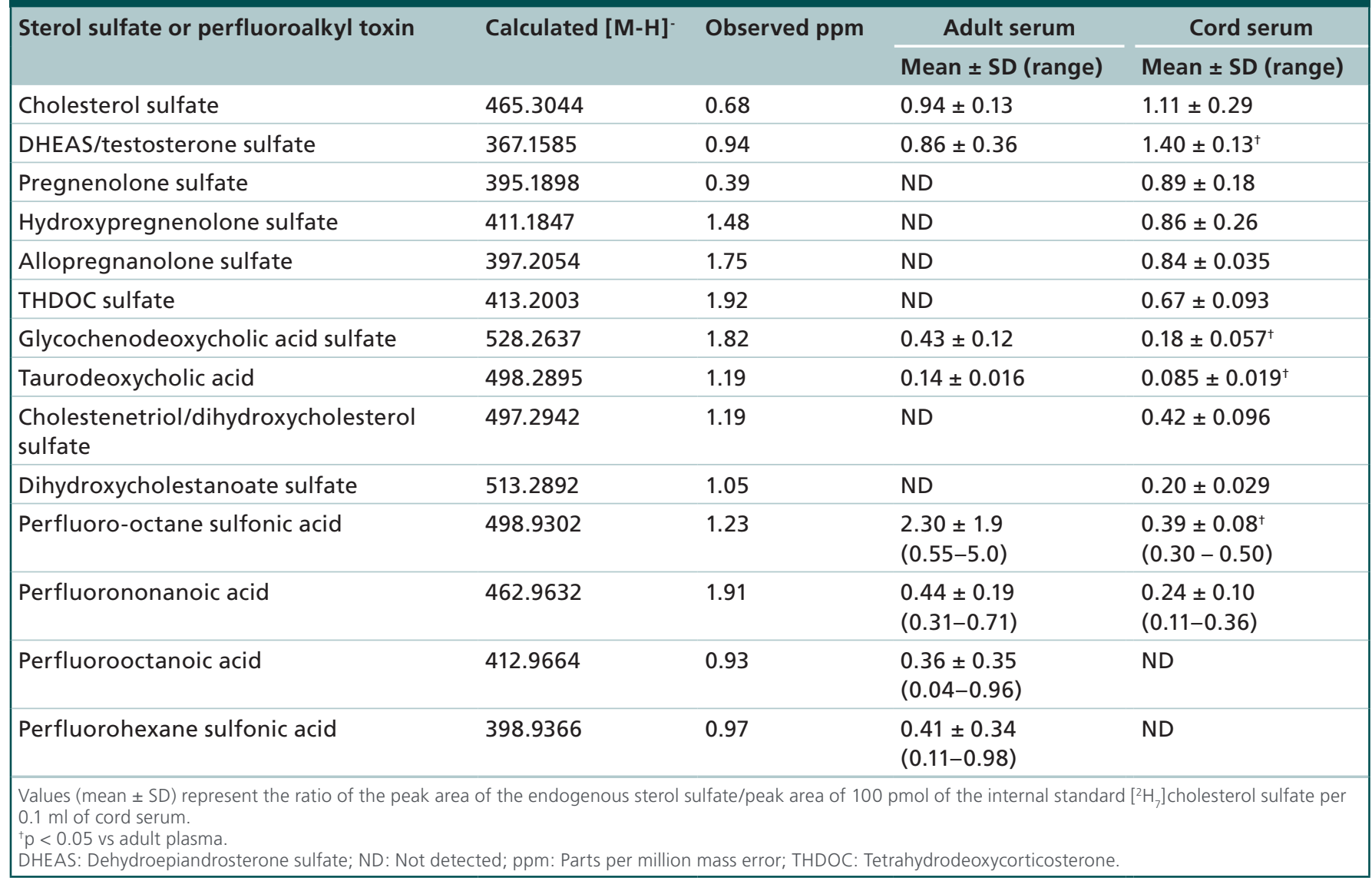

of these bile acid sulfates have been reported in infants with hydroxysteroid dehydrogenase deficiency [9-12] indicating that these may be useful biomarkers for abnormal fetal liver development.

Even though cord neurosteroids have been suggested to originate from the mother rather than the fetus [13-17], we observed that in the cord blood there were a number of neurosteroid sulfates which were absent in the adult sera. Supply of neurosteroids and neurosteroid sulfates is critical for the neurological development of the fetus, since they are potent modulators of GABAergic neuronal transmission [18,19] and are involved in synaptic plasticity and neuronal development [19-21]. It remains to be determined if the neurosteroid sulfates we monitored originate from the fetus or from the mother.

We confirmed previous findings that perfluoroalkyl-containing environmental toxins are present both in cord sera and in adult sera [22-26]. Analyzing cord blood for perfluoroalkyl toxins may be a useful means of determining fetal exposure to these carcinogenic environmental toxins, which have a detrimental effect on birthweight and sexual development of the fetus and young infant. The major limitation of this study is the small sample size. In addition, chromatographic methods should be utilized next to validate the identification of the sterols and perfluoroalkyl compounds.

\section{Conclusion}

In summary, our pilot study significantly expands our knowledge database for the lipidome of human cord sera and suggests that several potential biomarkers are worthy of further study. These include bile acid sulfates as potential biomarkers of abnormal fetal liver function, neurosteroid sulfates as potential biomarkers of fetal neural development and perfluoroalkyl-containing environmental toxins as potential biomarkers of fetal risks for slow development and early cancer development.

\section{Future perspective}

These data suggest that future studies of bile acid sulfates during in utero and postpartum development may lead to new diagnostics for evaluating normal versus abnormal liver development. Inclusion of pregnant and nonpregnant females also will be valuable comparisons in future studies. 


\section{Authors' contributions}

The authors contributed equally to the design, conduct and interpretation of the study.

\section{Financial \& competing interests disclosure}

This work was funded by College of Veterinary Medicine, Lincoln Memorial University, Sigrid Juselius Foundation and Finska Läkaresällskapet. The authors have no other relevant affiliations or financial involvement with any organization or entity with a financial interest in or financial conflict with the subject matter or materials discussed in the manuscript apart from those disclosed. This includes employment, consultancies, honoraria, stock ownership or options, expert testimony, grants or patents received or pending, or royalties.
No writing assistance was utilized in the production of this manuscript.

\section{Ethical conduct of research}

The authors state that they have obtained appropriate institutional review board approval or have followed the principles outlined in the Declaration of Helsinki for all human or animal experimental investigations. In addition, for investigations involving human subjects, informed consent has been obtained from the participants involved.

\section{Open access}

This work is licensed under the Creative Commons Attribution 4.0 License. To view a copy of this license, visit http://creativecommons.org/licenses/by/4.0/

\section{Executive summary}

- Sterol sulfates, including cholestenetriol and dihydroxycholestanoate sulfates, were detected in umbilical cord blood but not in the sera of the nonpregnant adult females, suggesting that bile acid metabolism in the fetus is more complex than in adults.

- Perfluoroalkyl toxins in umbilical cord blood may provide useful indices of the exposure of the fetus to these dangerous toxins.

\section{References}

Papers of special note have been highlighted as:

- of interest

1 Oresic M, Gopalacharyulu P, Mykkänen J et al. Cord serum lipidome in prediction of islet autoimmunity and Type 1 diabetes. Diabetes 62, 3268-3274 (2013).

2 La Torre D, Seppänen-Laakso T, Larsson HE et al. Decreased cord-blood phospholipids in young age-at-onset Type 1 diabetes. Diabetes 62, 3951-3956 (2013).

3 Wood PL, Scoggin K, Ball BA, Troedsson MH, Squires EL. Lipidomics of equine sperm and seminal plasma: Identification of amphiphilic (O-acyl)- $\Omega$-hydroxy-fatty acids. Theriogenology 86(5), 1212-1221 (2016).

- Overview of the high-resolution mass spectrometric lipidomics methodology utilized in this study.

4 Wood PL, Medicherla S, Sheikh N et al. Targeted lipidomics of fontal cortex and plasma diacylglycerols (DAG) in mild cognitive impairment and Alzheimer's disease: validation of DAG accumulation early in the pathophysiology of Alzheimer's disease. J. Alzheimers Dis. 48, 537-546 (2015).

5 Wood PL. Lipidomics of Alzheimer's disease: current status. Alzheimers Res. Ther. 4, 5 (2012).

- Overview of the complex lipid nomenclature.

6 Nardacci R, Falciatori I, Moreno S, Stefanini S. Immunohistochemical localization of peroxisomal enzymes during rat embryonic development. J. Histochem. Cytochem. 52, 423-436 (2004).

7 Seki Y, Matsushita M, Kimura A et al. Maternal and fetal circulation of unusual bile acids: a pilot study. Pediatr. Int. 53, 1028-1033 (2011).

- Historical data of cord bile acids (unsulfated).
8 Shoda J, Mahara R, Osuga T et al. Similarity of unusual bile acids in human umbilical cord blood and amniotic fluid from newborns and in sera and urine from adult patients with cholestatic liver diseases. J. Lipid Res. 29, 847-858 (1988).

9 Ichimiya H, Egestad B, Nazer H, Baginski ES, Clayton PT, Sjövall J. Bile acids and bile alcohols in a child with hepatic 3 beta-hydroxy-delta 5-C27-steroid dehydrogenase deficiency: effects of chenodeoxycholic acid treatment. J. Lipid Res. 32(5), 829-841 (1991).

10 Mizuochi T, Kimura A, Ueki I et al. Molecular genetic and bile acid profiles in two Japanese patients with 3betahydroxy-DELTA5-C27-steroid dehydrogenase/isomerase deficiency. Pediatr. Res. 68(3), 258-263 (2010).

11 Kimura A, Mahara R, Inoue $\mathrm{T}$ et al. Profile of urinary bile acids in infants and children: developmental pattern of excretion of unsaturated ketonic bile acids and 7betahydroxylated bile acids. Pediatr. Res. 45(4 Pt 1), 603-609 (1999).

12 Zhang W, Jha P, Wolfe B et al. Tandem mass spectrometric determination of atypical $3 \beta$-hydroxy- $\Delta 5$-bile acids in patients with $3 \beta$-hydroxy- $\Delta 5$-C27-steroid oxidoreductase deficiency: application to diagnosis and monitoring of bile acid therapeutic response. Clin. Chem. 61(7), 955-963 (2015).

13 Luisi S, Petraglia F, Benedetto C et al. Serum allopregnanolone levels in pregnant women: changes during pregnancy, at delivery, and in hypertensive patients. J. Clin. Endocrinol. Metab. 85, 2429-2433 (2000).

14 Hill M, Parízek A, Bicíková M et al. Neuroactive steroids, their precursors, and polar conjugates during parturition and postpartum in maternal and umbilical blood: 1. identification and simultaneous determination of pregnanoloneisomers. J. Steroid Biochem. Mol. Biol. 75, 237-244 (2000). 
- Historical data of neurosteroids (unsulfated) in cord blood.

15 Hill M, Bicíková M, Parízek A et al. Neuroactive steroids, their precursors and polar conjugates during parturition and postpartum in maternal blood: 2. Time profiles of pregnanolone isomers. J. Steroid Biochem. Mol. Biol. 78, 51-57 (2001).

16 Hill M, Parízek A, Klak J et al. Neuroactive steroids, their precursors and polar conjugates during parturition and postpartum in maternal and umbilical blood: 3.3betahydroxy-5-ene steroids. J. Steroid Biochem. Mol. Biol. 82, 241-250 (2002).

17 Luisi S, Petraglia F, Benedetto C et al. Serum allopregnanolone levels in pregnant women: changes during pregnancy, at delivery, and in hypertensive patients. J. Clin. Endocrinol. Metab. 85, 2429-2433 (2000).

18 Carter RD, Wood PL, Wieland S et al. Characterization of the anticonvulsant properties of ganaxolone (CCD 1042; $3 \alpha$-hydroxy-3 $\beta$-methyl-5 $\alpha$-pregnan-20-one), a selective, high-affinity steroid modulator of the $\gamma$-aminobutyric acid ${ }_{A}$ receptor. J. Pharmacol. Exp. Ther. 280, 1284-1295 (1997).

19 Fanelli F, Marino R, Keller F. Focusing on the interactions between the GABAergic system and neurosteroids in neurodevelopmental disorders. Curr. Pharm. Des. 19, 6491-6498 (2013).

20 Reddy DS. Neurosteroids: endogenous role in the human brain and therapeutic potentials. Prog. Brain Res. 186, 113-137 (2010).
21 Smith CC, Gibbs TT, Farb DH. Pregnenolone sulfate as a modulator of synaptic plasticity. Psychopharmacology (Berl) 231, 3537-3556 (2014).

22 Lien GW, Huang CC, Shiu JS et al. Perfluoroalkyl substances in cord blood and attention deficit/ hyperactivity disorder symptoms in seven-year-old children. Chemosphere 156, 118-127 (2016).

23 Chen MH, Ha EH, Liao HF et al. Perfluorinated compound levels in cord blood and neurodevelopment at 2 years of age. Epidemiology 24, 800-808 (2013).

24 Fisher M, Arbuckle TE, Liang CL et al. Concentrations of persistent organic pollutants in maternal and cord blood from the maternal-infant research on environmental chemicals (MIREC) cohort study. Environ. Health. 15, 59 (2016).

25 Sjogren P, Montse R, Lampa E et al. Circulating levels of perfluoroalkyl substances are associated with dietary patterns - a cross sectional study in elderly Swedish men and women. Environ. Res. 150, 59-65 (2016).

- Excellent overview of perfluoroalkyl toxins in human blood.

26 Romano ME, Xu Y, Calafat AM et al. Maternal serum perfluoroalkyl substances during pregnancy and duration of breastfeeding. Environ. Res. 149, 239-246 (2016). 